\title{
OPPORTUNITY FOR EARLY SCIENCE RETURN BY THE ARTEMIS PROĞRAM
}

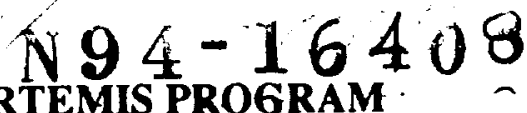

Charles Meyer, SN2, NASA Johnson Space Center, Houston TX

The purpose of the Artemis Program is to gather vital scientific and engineering data by conducting robotic exploration missions on the lunar surface both prior to and concurrent with human missions. The Artemis Program includes rapid, near-term development of a variety of small experimental and operational payloads, a low-cost capacity to deliver these payload to any location on the lunar surface, and the analysis of the data returned. The Artemis Program will provide opportunities to improve the understanding of lunar geosciences, to demonstrate the Moon's unique capacity as an astronomical platform to study the universe, to conduct scientific and technology development experiments, and to prepare for and complement human missions.

"Human beings have never yet put their footprints on a new land and then turned back, never to return." [1] However, the return to the lunar surface should include a partnership of robots and humans working together [2]. Robotic missions should explore numerous places on the Moon based on site selection using data collected by lunar polar orbiters and Earth-based telescopic observation. Rovers should be used to reconnoiter the landing area and paths to be followed by human explorers. Once a site for a lunar base is selected, rovers should be used to extend the reach and capability of astronauts. Multi-spectral imaging systems and various analytical instruments on rovers will allow close-up observations of outcrops and preliminary selection of important samples to be returned to Earth. Although, human field geologists may be required to make the final selection of samples to be returned, well-equipped rovers could make an informed preliminary examination of numerous samples collected from a wide area over a long period of time. Astronauts working out of a lunar base would then be able to concentrate their time on the most important outcrops. Rovers could also be used to deploy, align and attend geophysical and astronomical instruments as well as pilot plants for "resource" development. In general, tasks that can be accomplished by robots should not be done alone by humans.

Scientific goals for future robotic lunar geoscience lander missions are; 1) to develop a more complete understanding of the stratigraphy, structure, composition and evolution of the lunar crust by close examination of the geology and geochemistry of multiple, carefully-selected, wide-spaced landing sites on the Moon; 2) to improve the understanding of the lunar regolith and history of solar system events that have affected the lunar surface; 3 ) to improve the understanding of the lunar interior and set constraints on planetary evolution using geophysical techniques; 4) to identify and characterize potential lunar "resources" that could be utilized by future human missions; and 5) to identify and transport important samples to a (future) sample return vehicle. A high degree of mobility is a primary requirement for lunar geoscience landers. Chemical and mineralogical analyses are required for hundreds of samples, including rock fragments, collected over hundreds of kilometers. Exploration of the Moon using scientific rovers would also provide additional engineering data and confidence for mission planners for the use of similar devices on Mars and provide significant educational and training opportunities for the next generation of space explorers and robotics engineers.

Scientific rovers should be designed as integrated scientific measurement systems. There is currently much enthusiasm for the use of rovers in planetary exploration [3], but scientific goals (above) must remain the focus of this enthusiasm and all of the instruments, cameras and high-fidelity, supporting elements must work together as a system. Scientists experienced with the study of samples returned by Apollo will demand that rocks be studied as well as regolith samples and that certain key elements be measured with precision. The Russian Lunokhod has already demonstrated the engineering feasibility of wide-ranging mobility on the lunar surface, however, future lunar rovers should be able to travel several hundred kilometers and last several lunar nights. One of the current programmatic problems that must be overcome, is that the development of analytical instrumentation for use on planetary surfaces has been sadly neglected for many years. A light-weight alpha and proton backscatter spectrometer is flight-ready and a backscatter Mossbauer spectrometer is under development. However, additional instrument development is essential. Light-weight, CCD-based multi-spectral imaging systems, covering 
ARTEMIS PROGRAM: Charles Meyer

the spectrum from 0.3 to 2.5 microns, are the result of an emerging technology that will become available, but auto-focus lenses for these cameras need to be developed for the cold lunar environment. Manipulator arms, close-up cameras and supporting systems would make a true robotic field geologist! With these instruments and tools, a highly mobile rover will be able to measure the chemical and mineralogical composition and texture of samples in situ. Each Artemis rover should also deploy a small seismograph, such as designed for the MESUR missions, a short distance from the lander, until there is a global seismic network on the Moon.

Previous study of the Moon has focused on the small area of the Apollo and Luna landings. There is evidence that rock types might be quite different at distant locations on the Moon. The new geochemical data from Galileo [4], and expected from Clementine and Scout, will allow us to target specific geochemical regions that will require "ground truth" from robotic missions and eventual sample return. From Apollo we have learned that the large basins on the front side have covered the northem highlands with thick sheets of "gray ejecta." However, Earth-based telescopic studies now show a number of places in the southern highlands, where there are apparently windows through the basin deposits of gray ejecta [5], that should be investigated by highly-instrumented scientific rovers.

The Moon has several advantages as a potential site for astronomical observations as well as several apparent disadvantages (cold, dusty environment) that need to be explored and overcome. The lack of essentially any lunar atmosphere allows superb viewing conditions in the ultraviolet wavelengths and several proposals have been made for a sky survey in the UV by small robotic lunar telescopes operating around $200 \mathrm{~nm}$. This concept was already proven during Apollo 16, but the azimuth tracking mechanism partially froze in the shade of the LM requiring astronaut intervention. Since experience is the great teacher, numerous other, small precursor telescopes should be attempted before large lunar telescopes are assembled by human missions. The Moon itself has very low seismic activity such that it may serve as an ideal platform for long-baseline interferometry. However, the extreme thermal variations make design of stable telescopes a difficult proposition. Experimentation would seem appropriate. Since the Moon always points towards the Earth, the Moon would be an ideal site for extreme ultraviolet imaging of transitory phenomena associated with the solar wind interaction with the Earth's outer magnetosphere. In this way, long-term, synoptic viewing of the Earth's magnetosphere at various wavelengths in the EUV (30 nm) would allow a study of variations in the corpuscular radiation of the Sun and test models of the Earth's (and other planets) magnetosphere(s).

The extraction of oxygen from lunar rocks has been suggested as a potential "resource" for human missions to the Moon and beyond. Ilmenite-rich soils and/or iron-rich glass deposits have been shown to have the highest yield of $\mathrm{H}_{2} \mathrm{O}$ in a hydrogen furnace. Robotic missions should be used to locate these deposits and to test pilot plant operations prior to human missions. Depending on the results achieved by experiments performed on robotic missions, the scenario for the first lunar outpost may be changed.

JSC has designed a Common Lunar Lander for the Artemis Program based on a planned payload weight of $200 \mathrm{~kg}$. This weight was selected to meet the requirements for large scientific rovers, small $(0.8 \mathrm{~m})$ telescopes, pilot plants and even for potential sample return in the future. Under present scenarios, the Artemis lander could land $85 \mathrm{~kg}$ using a Delta II or $200 \mathrm{~kg}$ using an Atlas launch vehicle. In the JSC design, no payload services will be provided by the lander Atlas launch vehicle. In the JSC design, no payload services will be provided by the lander
vehicle, but it will be able to safely land a payload anywhere on the lunar surface (within $3 \mathrm{~km}, 3$ sigma). The top of the landed vehicle will be about 60 inches above the lunar surface. This newly-designed spacecraft makes use of several recent technology developments including; graphite-epoxy structure, fiber-overrapped tanks, SDI light-weight, high-performance engines, a new range velocity sensor, a new light-weight Startracker and a central avionics architecture. The Common Lunar Lander could be flight-ready in about 3.5 years from start.

References: [1] Heiken G., Vaniman D. and French B. (1991) The Lunar Sourcebook: a users guide to the moon, Cambridge Univ. Press, p640. [2] U. S. Congress, O.T.A. (1991) Exploring the Moon and Mars: Choices for the Nation, U.S. Gov. Printing Office. [3] see for example, Planetary Report Nov/Dec 1992; Final Frontier Feb 1993. [4] Belton M. et. al. (1992) Lunar Impact Basins and Crustal Heterogeneity, Science 255, 570. [5] Hawke B et al. (1992) The Distribution and Modes of Occurrence of Lunar Anorthosite: abs. LPSC XXIII, 505, LPI. 